Voix et Images

volxetimages

\title{
Narrativités minimalistes contemporaines
}

Toussaint, Tremblay, Turcotte

Contemporary Minimalist Narrativities

Toussaint, Tremblay, Turcotte

Narratividades minimalistas contemporáneas

Toussaint, Tremblay y Turcotte

\section{Marie-Pascale Huglo et Kimberley Leppik}

Volume 36, numéro 1 (106), automne 2010

Narrations contemporaines au Québec et en France : regards croisés

URI : https://id.erudit.org/iderudit/045233ar

DOI : https://doi.org/10.7202/045233ar

Aller au sommaire du numéro

\section{Éditeur(s)}

Université du Québec à Montréal

\section{ISSN}

0318-9201 (imprimé)

1705-933X (numérique)

Découvrir la revue

\section{Citer cet article}

Huglo, M.-P. \& Leppik, K. (2010). Narrativités minimalistes contemporaines : Toussaint, Tremblay, Turcotte. Voix et Images, 36(1), 27-44.

https://doi.org/10.7202/045233ar

\section{Résumé de l'article}

Le discours critique fait du minimalisme narratif une caractéristique du contemporain en France comme au Québec. Marque par excellence d'un retour à la lisibilité et au récit, le minimalisme, dans quelques-unes de ses formes marquantes, relève pourtant d'une sorte de malaise : les réductions de tous ordres, la disparition d'enjeux d'envergure et la dilution de la littérature dans un univers médiatique éclaté font de cette tendance un

" amoindrissement ", un " repli " plus ou moins nostalgique. Si les contextes historiques et culturels du Québec et de la France diffèrent, la critique tend de part et d'autre à associer la " réduction " minimaliste à la perte d'envergure de la littérature contemporaine, qui raconterait " malgré tout », que ce soit sur un mode fragmentaire, intimiste ou ludique. Nous proposons de revenir sur cette conception, de faire travailler les convergences et divergences critiques entre la France et le Québec pour tenter de cerner une certaine narrativité minimaliste, distincte de ce qu'on appelle parfois « le mode mineur ». Nous nous basons sur une lecture de Jean-Philippe Toussaint, Larry Tremblay et Elise Turcotte pour remettre en question l'idée d'une réduction minimaliste et reconsidérer le rapport entre le sensible et le sens, en insistant sur l'importance, peu soulignée jusqu'ici, de la tension narrative et des imaginaires qui mobilisent ce type de récit. 


\title{
N A R R A T I V I T ÉS \\ M I N IM A LISTES CONTEMPOR A INES.
}

Toussaint, Tremblay, Turcotte ${ }^{1}$

$+++$

\author{
MARIE-PASCALE HUGLO \\ Université de Montréal \\ KIMBERLEY LEPPIK \\ Université de Montréal
}

Pour qui cherche à cerner le minimalisme, le caractère nébuleux de la notion sème une certaine confusion. Alors que Sophie Deramond s'efforce de le définir à partir d'Un an de Jean Echenoz ${ }^{2}$, Jan Baetens, dans un article paru dans le même numéro d'Intervalles consacré aux "Minimalismes», affirme que "seul un tenace malentendu, dû aux seuls aléas de la publication, continue à ranger [Jean Echenoz] parmi l'école minimaliste ${ }^{3}$ ». Ce manque de cohérence est perceptible dans toute la critique sur le minimalisme contemporain en France et au Québec. Sans vouloir à tout prix clarifier une notion qui, manifestement, s'affirme dans un flou conceptuel et critique, nous proposons, dans un premier temps, de faire le tour de quelques approches théoriques françaises et québécoises afin d'en dégager des caractéristiques significatives. Nous passerons ensuite à l'analyse d'ouvrages représentatifs de certains courants minimalistes des deux littératures afin d'évaluer les particularités formelles et thématiques qui les éloignent et les rapprochent. Enfin, nous tenterons, sur la base de cette étude comparée, de définir la narrativité minimaliste à la croisée d'une esthétique et d'une poétique du récit.

$$
+++
$$

1 Cet article fait partie du projet «Littératures narratives contemporaines française et québécoise. Poétiques et esthétiques comparées » subventionné par le Fonds québécois de la recherche sur la société et la culture (FQRSC). 2 «Dans la littérature française contemporaine, une certaine confusion demeure : le qualificatif de "minimaliste" se trouvant appliqué tantôt à Philippe Delerm et à certains de ces écrivains du quotidien que Pierre Jourde dénonce dans La littérature sans estomac [Paris, Esprit des péninsules, coll. «L'alambic», 2002, 333 p.], tantôt à une nébuleuse d'écrivains aussi divers que Hervé Guibert, Annie Ernaux, Leslie Kaplan, Pierre Michon, JeanPhilippe Toussaint, Jean Echenoz, Antoine Volodine, etc. Une autre vision plus ou moins admise par la critique et le milieu universitaire considère le groupe plus restreint des jeunes auteurs des Éditions de Minuit comme les seuls représentants sûrs d'une école minimaliste française. " Sophie Deramond, "Un an de Jean Echenoz", Intervalles, vol. I, $\mathrm{n}^{\circ}$ 1, automne 2004, p. 125-126. 3 Jan Baetens, «Pour en finir avec la poésie dite minimaliste», Intervalles, vol. I, nº 1, automne 2004, p. 104. 


\section{LE Minimalisme DE MinUit : UNE NARRATIVité RÉdUite}

Pour éviter un usage trop «lâche» de la notion, nous limiterons notre étude du minimalisme français contemporain à la réception des jeunes auteurs de Minuit, un groupement d'écrivains dont la périodisation se révèle d'emblée, elle aussi, délicate. On constate en effet une importante disparité de la critique. Ces auteurs et leurs œuvres ont pu, par exemple, recevoir une pléthore d'étiquettes et de classifications : «le clan des éditions de Minuit», "la jeune ou la nouvelle génération de Minuit», «une tendance littéraire», "une orientation", «un courant plutôt qu'une école», «la génération salle de bain», les "romans impassibles ${ }^{4} »$. Une décennie après le tournant du millénaire, force est de constater que ces «jeunes auteurs» ont vieilli, que leurs œuvres ont évolué de manière, peut-être, à infléchir la compréhension critique de cette mouvance qui a fait sa marque et se poursuit désormais à distance du nouveau roman et de "l'ancienne génération de Minuit». C'est précisément cette distance que nous voulons investir pour reconsidérer cette notion au-delà des effets de nouveauté.

Dans Small Worlds, Warren Motte fait du "petit», c'est-à-dire d'une réduction en relation avec une norme plus ou moins explicite, le dénominateur commun de toutes les formes du minimalisme dans l'art contemporain ${ }^{5}$. Motte est le premier à souligner la relativité d'un tel critère, qui se détermine par rapport à une «norme» qui reste à définir. Il fait dériver l'étiquette littéraire de l'art et de la musique minimalistes, mais il se retrouve dans une impasse. Le minimalisme dans les arts visuels et dans la musique cherche en effet à éviter toute représentation narrative, se concentrant sur le "thingness», sur la matérialité de l'objet. Pourtant, comme Motte lui-même le souligne, la littérature est nécessairement représentative et comporte, fût-ce minimalement, une dimension narrative. Le déplacement est d'autant plus notable qu'alors que le minimalisme en art entend d'abord rompre avec la représentation dans une logique avant-gardiste, le minimalisme de Minuit est présenté comme l'une des manifestations du retour à la narrativité et de la fin des avantgardes dans la culture contemporaine. La critique littéraire aurait donc contribué à faire du minimalisme une manifestation distinctive du régime contemporain des «retours». En résulte une définition plus souple qui pose problème: si le minimalisme sous toutes ses formes réengage une narrativité tout en la réduisant, reste à savoir de quelle réduction il s'agit.

$$
++
$$

4 Fieke Schoots, «Passer en douce à la douane». L'écriture minimaliste de Minuit: Deville, Echenoz, Redonnet et Toussaint, Amsterdam, Rodopi, coll. «Faux titre», 1997, p. 16. 5 Warren Motte, Small Worlds. Minimalism in Contemporary French Literature, Lincoln, University of Nebraska Press, 1999, p. 1. Notons ici que le corpus que Warren Motte rassemble dans son étude sur le minimalisme en France confirme le flou problématique de l'étiquette: à côté de Jean-Philippe Toussaint et Marie Redonnet (généralement rangés parmi les minimalistes), il choisit d'inclure des écrivains aussi divers que Marie NDiaye, François Bon, Hervé Guibert, Eugène Savitzkaya (tous sont considérés par Fieke Schoots comme des écrivains non minimalistes [ "L'écriture "minimaliste" ", Michèle Ammouche-Kremers et Henk Hillenaar (dir.), Jeunes auteurs de Minuit, "CRIN", n 27, 1994, p. 127144]) et Antoine Volodine, écrivain que Lionel Ruffel, pour sa part, qualifie de "maximaliste» (Le dénouement, Lagrasse, Verdier, coll. «Chaoïd», 2005, 106 p.). 
Bien répandue dans la critique sur les auteurs minimalistes est l'idée qu'ils sont entravés par ceux qui les ont précédés et qu'ils ne peuvent écrire que dans un après, malgré le soupçon. Dans son article "Écrire au présent. L'esthétique contemporaine», Dominique Viart affirme que la «nouvelle école de Minuit» fait des «fictions réduites ${ }^{6}$ » et que ses narrateurs «impassibles», avec leur ton "désabusé et légèrement ironique», sont caractéristiques de ce qu'Alain Nadaud appelle le "désenchantement». Selon Viart, leurs romans «jouent de modèles romanesques sans jamais leur donner l'ampleur ni l'intensité qu'un lecteur accoutumé à ces modèles est en situation d'attendre ${ }^{7}$ ». Il note l'atemporalité de ces récits et remarque qu'ils se concentrent sur la «simple facticité» des choses. Ils lui semblent «ne mettre en scène qu'une pure pulsion narrative, mais sans matière de récit, sans véritables histoires dignes d'être romancées ${ }^{8}$ ». La réduction narrative s'inscrirait donc par rapport à un horizon romanesque à grand déploiement; elle irait même jusqu'à fragiliser l'histoire dont les auteurs reconduiraient la manière sans en conserver toute l'étoffe (action, personnages, intrigue). Viart voit dans cette réduction «la manifestation d'une nostalgie par défaut: le désir même de "continuer à écrire" exprime le regret tacite de la Littérature (avec majuscule) ${ }^{9}$ ".

Viart considère que ces écrivains ont choisi l'«option ludique» plutôt que l'«option naïve» pratiquée par les romanciers de la nouvelle fiction, ludisme qui ressort comme un trait dominant du minimalisme en France, où l'ironie et l'humour impassible se dessineraient sur le fond $d^{\prime}$ une perte ${ }^{10}$. Mais si tout écrivain doit écrire «après», si le soupçon ${ }^{11}$ reste une des manières d'envisager l'émergence paradoxale du roman minimaliste ${ }^{12}$, la critique ne limite pas cette mouvance à une tentative d'écrire malgré tout, presque par défaut. C'est Renaud Camus qui souligne que le romancier contemporain hérite «de toutes les contraintes formelles de ses prédécesseurs et d'une contrainte supplémentaire, qui [est] le devoir d'être lisible ${ }^{13}$ ».

Ainsi, l'apparente simplicité qu'on croit déceler dans ces récits est l'effet d'un travail d'écriture qui réduit systématiquement les techniques rhétoriques (ce qui est, en soi, une technique rhétorique...) et réorganise le récit autour de principes dissimulés aux yeux du lecteur. L'art devient chez ces écrivains un jeu de cachecache, redoublé par le ton ludique et des contraintes «formelles ou chiffrées » qui renforcent leur désinvolture manifeste ${ }^{14}$. Ce minimalisme ne se dissocie donc pas d'un souci formaliste ni même d'une forme de littérarité ${ }^{15}$; il ne rompt pas avec

$$
++
$$

6 Dominique Viart, «Écrire au présent. L'esthétique contemporaine», Michèle Touret et Francine Dugast-Portes (dir.), Le temps des lettres. Quelles périodisations pour l'histoire de la littérature française du $20^{\circ}$ siècle?, Rennes, Presses universitaires de Rennes, coll. «Interférences», 2001, p. 323.7 Dominique Viart, art. cité, p. 329. 8 Dominique Viart, art. cité, p. 329. 9 Dominique Viart, art. cité, p. 329. 10 Dominique Viart, art. cité, p. 330. 11 Écrire après l'ère du soupçon, c'est écrire avec le soupçon. Selon Jan Baetens, «le minimalisme [...] relève [...] d'un effort, souvent pénible et maladroit, de réinventer le récit romanesque après l'ère du soupçon» («Pour en finir avec la poésie dite minimaliste», p. 104). 12 La mesure de l'amplitude romanesque est, certes, délicate à établir, mais en admettant que la complexité et le déploiement sont, d'un point de vue pragmatique, ce qui démarque le roman d'autres genres narratifs en ce qui concerne la temporalité, les personnages et l'action, on peut ainsi considérer l'étiquette de roman minimaliste comme intrinsèquement paradoxale. 13 Cité dans Yvan Leclerc, "Autour de Minuit», Dalhousie French Studies, vol. 17, automne-hiver 1989, p. 67. 14 Yvan Leclerc, art. cité, p. 67-68. 15 En ce sens, le minimalisme de Minuit se distingue d'un minimalisme volontairement pauvre, plat, tournant le dos à la belle langue comme à l'illusion romanesque 
l'illusion de la mimèsis mais la reconduit au second degré, dans des fables sans enjeux apparents.

De toutes les analyses théoriques ayant traité des jeunes auteurs de Minuit, il nous semble que celle de Fieke Schoots est la plus fouillée. Selon elle, ces écrivains pratiquent un minimalisme narratif qui fonctionne sur trois aspects ${ }^{16}$. Sur le plan de la forme, on constate la brièveté des mots, des phrases, des fragments, des paragraphes, des récits et des œuvres; en ce qui a trait au style, la syntaxe et le vocabulaire sont simplifiés et imprécis ${ }^{17}$, les conjonctions de subordination sont rares, la langue "parlée» mène souvent à des phrases inachevées ou grammaticalement incorrectes; pour ce qui est du contenu, l'intrigue, les personnages et le décor sont réduits, les relations de causalité entre les événements sont absentes et, pour finir, l'écoulement du temps est dissimulé.

Fieke Schoots souligne également d'autres caractéristiques méritant d'être notées. La surabondance d'intertextualité, qui convoque non seulement la littérature mais aussi les arts visuels - qu'ils soient nobles (peinture, cinéma) ou moins nobles (télévision, bande dessinée) -, est l'indice d'un «jeu citationnel» poussé. Si l'omniprésence de tels renvois se distance d'une représentation du monde naïve en la projetant comme artifice, elle est aussi ce qui, par la multiplication des images, «tend à effacer les frontières entre la réalité et la fiction » et rend manifeste «le rôle de l'art ou de la représentation dans les rapports que l'homme entretient avec le monde ${ }^{18}$ ». Parallèlement, un « réenchantement du monde ${ }^{19}$ » est opéré par le jeu langagier et le ton ludique. Ainsi, les minimalistes «soulignent encore une fois que la vision du monde passe forcément par la représentation ${ }^{20}$ ». Enfin, le refus de tout rapport traditionnel entre cause et effet (sur le plan syntaxique comme sur celui de l'intrigue) est ce qui organise, d'une certaine manière, le récit. Ce dernier reste cohérent malgré sa forme fragmentaire; on décèle une continuité événementielle à travers les blancs formels, syntaxiques et narratifs qui le traversent et le charpentent. La ligne de fuite du récit comme principe organisateur, couplée avec le jeu citationnel et le réenchantement du monde, déréalisent le réel sans que les auteurs renoncent pour autant à se «prononcer ${ }^{21}$ » sur la réalité. Ce faisant,

les "minimalistes" français [...] "déréalisent" le réel, de sorte que le récit se présente comme une représentation. [...] L'originalité de l'écriture minimaliste réside donc

$$
+++
$$

caractéristiques de la littérature dominante. L'œuvre d'Annie Ernaux, à partir de La Place, est la plus représentative, en France, de ce courant, dont la visée réaliste et les enjeux sociaux se dressent contre la grande tradition littéraire sans un soupçon de nostalgie, loin des errances apparemment sans portée des Impassibles. En ce qui a trait à la narrativité, on notera cependant, dans les deux cas, l'importance du modèle romanesque, qu'on le détourne (aplatissement diégétique et second degré) ou qu'on s'en détourne (rejet de la fiction et de l'artifice littéraire). 16 Fieke Schoots, "Passer en douce à la douane», p. 52-55. 17 Schoots souligne l'utilisation fréquente de formulations telles que "assez grand», «assez loin», «le surlendemain», etc., qui n'ont pas de signifiance précise sauf en relation avec d'autres mots, souvent manquants dans les textes. 18 Fieke Schoots, "Passer en douce à la douane», p. 183. 19 Fieke Schoots, "Passer en douce à la douane», p. 184. 20 Fieke Schoots, "Passer en douce à la douane», p. 184. 21 Nous paraphrasons ici: "grâce au jeu langagier, la réalité s'y déréalise»; ; [Les minimalistes] ne s'en prononcent pas moins sur la réalité». Fieke Schoots, «Passer en douce à la douane», p. 184. 
dans sa façon de redécouvrir le récit tout en mettant en cause la représentation de la réalité par le langage. [...] D'une part, l'écriture minimaliste poursuit donc le travail subversif de l'avant-garde minimaliste, d'autre part elle redécouvre les éléments narratifs traditionnels rejetés par cette même avant-garde ${ }^{22}$.

Le minimalisme de Minuit est difficile à cerner, on le voit. Cet aperçu fait ressortir à quel point les caractéristiques narratives qu'on lui rattache peuvent être reçues par la critique dans des sens opposés. La réduction impliquée par l'étiquette du «minimalisme» n'est pas un critère esthétique stable et entraîne un jugement de valeur variable. Dans le sens de "fictions réduites", la facticité et l'atemporalité sont, pour certains critiques, considérées comme un défaut de matière et de profondeur romanesque. Même le ton ludique et l'humour impassible impliquent un soupçon vis-à-vis des "grands moyens» de l'esthétique romanesque, auxquels il ne serait plus question de recourir naïvement. La lisibilité et la narrativité retrouvées semblent dès lors le signe d'une vacuité, d'une nostalgie, d'un malgré tout qui, en douceur et de manière désinvolte, remet la représentation en cause. Pourtant, ce qui semble désenchanté ici est considéré, là, comme un réenchantement: le jeu affiché avec le roman et les autres arts est alors perçu comme une qualité capable de relancer la question du sens. Cette relance s'énonce, toutefois, de manière ambiguë. Fieke Schoots maintient ainsi l'idée d'une réduction tout en insistant, dans son bilan, sur ce que celle-ci suppose: si, pour elle, le second degré des écrivains de Minuit compromet la représentation, elle insiste aussi sur la prise de conscience des moyens de la représentation que cette pratique engage. L'autre élément à relever est l'abolition de l'enchaînement de cause à effet - autrement dit d'une forme canonique de l'intrigue centrée sur l'action -, l'attention portée aux manifestations de surface et l'absence d'ancrage temporel comme principes organisateurs du récit auxquels répondent, selon Fieke Schoots, d'autres principes d'organisation, d'ordre formel ${ }^{23}$.

\section{LE MINIMALISME ET LE MODE MINEUR DANS LA LITTÉRATURE QUÉBÉCOISE CONTEMPORAINE}

Du côté québécois, le minimalisme est également considéré comme caractéristique de la littérature contemporaine. Sous cet étendard du moins, il permet de conjoindre des écrivains très différents. Si l'on s'en tient aux fictions narratives, il est là encore associé à la fin d'une époque au sortir de laquelle émerge, à partir des années 1980, le «contemporain». Tout comme en France, le minimalisme correspond à une expansion

$$
++
$$

22 Fieke Schoots, «Passer en douce à la douane», p. 57-58. 23 Schoots désigne l'arbitraire, le hasard, la réflexion chaotique et la récursivité comme possibles principes organisateurs du récit minimaliste. Cela va dans le sens de l'écriture du ressassement, que Dominique Rabaté aborde comme un des traits caractéristiques de la littérature contemporaine (Éric Benoit, Michel Braud, Jean-Pierre Moussaron, Isabelle Poulin et Dominique Rabaté, Écritures du ressassement, Bordeaux, Presses de l'Université de Bordeaux, coll. «Modernités», 2002, 327 p.). 
littéraire "à l'ère du pluralisme ${ }^{24}$ », en l'absence de rupture, de révolution, de figures de proue, d'école littéraire ou de grands auteurs. Le changement se fait en douceur et il correspond à une perte, dans une période où la littérature "ne parvient pas à se représenter positivement, comme si elle était privée de repères ou ne se voyait que sur un mode négatif, en accumulant les signes de ce qu'elle a perdu, de ce qu'elle n'est plus ${ }^{25} »$. Michel Biron, François Dumont et Élisabeth Nardout-Lafarge soulignent, dans leur Histoire de la littérature québécoise, ce qui, dans la critique en France, s'inscrit en filigrane: il s'agit d'une perte symbolique liée au décentrement de la littérature nationale, à la pluralisation des identités culturelles, mais aussi à la marginalisation de la littérature comme telle par des médias comme «la radio, le cinéma, la télévision et [...] Internet ${ }^{26}$ ». D'un côté comme de l'autre, la littérature contemporaine émerge sur le fond d'une perte symbolique que la perspective historique de Biron, Dumont et Lafarge fait, pour le Québec, bien ressortir. Dans ce contexte, le minimalisme, avec la réduction qu'il implique, se situe de façon ouverte par rapport au grand récit national et collectif et aux expérimentations formalistes des années 1960-1970. La période contemporaine se trouve dès lors associée à l'expansion et à la pluralisation d'une petite littérature certes désormais florissante, mais décentralisée, sans enjeux majeurs. L'intimisme apparaît comme un trait dominant du minimalisme, répondant à la décomposition des enjeux collectifs par une exploration du sujet, quand «le mouvement vers soi» épouse un mouvement «vers le monde concret des choses et l'univers des perceptions immédiates ${ }^{27}$ ». Le roman «en mode mineur», représenté par Jacques Poulin, Louis Gauthier, Yvon Rivard ou Élise Turcotte, est, d'un point de vue formel, assez bref, répétitif, atténué quant aux conflits. D'un point de vue thématique, il se concentre sur l'individu et le quotidien dans un climat d'indétermination. Le rejet du formalisme et du déploiement romanesque au profit d'une écriture sobre, simple, introspective, toute en retenue, se dégage globalement de cette tendance qui croise, avec l'œuvre d'Aki Shimazaki par exemple, l'écriture migrante. Bien démarqué tant des expérimentations formelles et langagières de la génération précédente que des romans baroques, des romans d'action ou des best-sellers, le minimalisme québécois va dans le sens d'une lisibilité accrue. L'accent mis sur la subjectivité et l'intimisme, abordés avec gravité et profondeur, dans un parcours intériorisé, rattache ce mode à une exploration intime du réel; il se trouve aux antipodes du ludisme, de l'impassibilité et de la superficialité relevés chez les minimalistes de Minuit.

Un constat semblable ressort à la lecture de l'article de Frances Fortier et Andrée Mercier sur le minimalisme narratif dans les récits québécois avec, toutefois, des divergences notables ${ }^{28}$. Les récits minimalistes (qui, partant, se distinguent des romans) désignent « une limite inférieure ${ }^{29}$ » de la narrativité rompant avec l'enchaî-

$$
+++
$$

24 Michel Biron, François Dumont et Élisabeth Nardout-Larfage, Histoire de la littérature québécoise, Montréal, Boréal, 2007, p. 531.25 Michel Biron, François Dumont et Élisabeth Nardout-Larfage, ouvr. cité, p. 531. 26 Michel Biron, François Dumont et Élisabeth Nardout-Larfage, ouvr. cité, p. 534. 27 Michel Biron, François Dumont et Élisabeth Nardout-Larfage, ouvr. cité, p. 550. 28 Frances Fortier et Andrée Mercier, «La narration du sensible dans le récit contemporain", René Audet et Andrée Mercier (dir.), La narrativité contemporaine au Québec, Québec, Presses de l'Université Laval, 2004, p. 173-201. 29 Frances Fortier et Andrée Mercier, art. cité, p. 179. 
nement causal des événements. L'absence de perspective totalisante s'accompagne d'une forte visualité donnant le monde à voir sans l'expliquer. En cela, de nombreux récits minimalistes québécois et français convergent sur le plan esthétique. Les premiers se démarquent par la prédominance de la subjectivité intime, considérée par Fortier et Mercier moins comme un «thème» que comme le fondement d'une logique narrative basée sur le sensible, c'est-à-dire sur les émotions éprouvées par une conscience subjective. Cette logique du sensible permet de sortir du modèle narratif basé sur l'action pour épouser une autre logique, celle de la passion, qu'elle infléchit en la dégageant des excès attendus pour aller du côté d'affects moins intenses, plus ténus, plus finement modulés ${ }^{30}$. Ainsi, de manière fragmentaire, les récits minimalistes se limitent à une "saisie impressive" sans orchestration d'ensemble qui, en déjouant la mise à distance réflexive et temporelle, met de l'avant une subjectivité en prise immédiate avec le monde. L'intimisme subjectif minimal est alors le siège de sensations et de perceptions qui font événement sans nécessairement faire sens. On retrouve ici l'insignifiance des romans de Minuit mais, tandis que du côté français, on l'associe à une superficialité sans profondeur, elle relève, du côté québécois, d'une sensibilité subjective immédiate, sans perspective. Les jeux intertextuels et le ton impassible, tout en ruptures, des voix narratives «de Minuit» n'ont manifestement rien à voir avec une sensibilité que l'on pourrait croire naïve tandis que, dans le rejet de l'intrigue et la fragmentation impressive de la vision du monde, les deux minimalismes convergent.

Le rejet de l'intrigue - avec la perspective totalisante, l'enchaînement causal, l'orchestration des personnages et de l'action, l'orientation temporelle et la visée qui s'y rattachent - se trouve, dès lors qu'il se combine avec le maintien d'une histoire lisible, au cœur de la narrativité minimaliste. Cela constitue un critère commun certes large mais suffisant, sans pour autant préciser ce qui motive le maintien du récit. Les étiquettes de minimalisme et de mode mineur encouragent une lecture critique axée sur la réduction ou l'atténuation, et tendent à faire perdre de vue ce qui déstabilise et déplace les attentes narratives dans un geste déroutant. Dans la perspective comparatiste qui est la nôtre, il importe de savoir si l'impassibilité ludique sur laquelle s'est construite la réputation des écrivains de Minuit et l'intimisme revendiqué pour les minimalistes québécois, d'une part sont aussi divergents qu'on le suppose et, d'autre part, peuvent être considérés comme partie prenante d'une même approche narrative d'ensemble.

$$
++
$$

30 Précisons ici que Fortier et Mercier s'appuient sur l'approche sémiotique de Fontanille, pour qui le devenir narratif se décline suivant trois logiques qui se combinent entre elles: l'action, la cognition et la passion. La logique du sensible «minimalise» le modèle passionnel tout en récusant l'idée largement reconnue que l'action finalisée est la seule mesure du changement à la base du devenir narratif. Frances Fortier et Andrée Mercier, art. cité, p. 182. 
S'il est vrai que la critique manque de cohérence dans sa définition du minimalisme littéraire en France, certains consensus demeurent. C'est le cas du jugement émis à propos de l'œuvre de Jean-Philippe Toussaint, qui, d'après Dominique Viart ${ }^{31}$, Fieke Schoots ${ }^{32}$ et Jan Baetens ${ }^{33}$, doit impérativement être rangée sous cette bannière. Cette classification s'explique d'abord par le critère éditorial: Toussaint est bel et bien un «jeune auteur de Minuit». Mais il y a plus. Le roman La salle de bain, paru en 1985, donc en amont d'une périodisation que certains feraient débuter en $1989^{34}$, est considéré comme précurseur de la mouvance esthétique ${ }^{35}$. Avec Toussaint, il semble bien que nous ayons affaire au parangon du minimalisme, dont La salle de bain constituerait le coup d'envoi: l'absence d'intrigue, les personnages et les espaces restreints, la relative brièveté du récit qui caractérisent ce roman vont dans ce sens. Mais le minimalisme n'y est pas aussi évident en ce qui concerne l'écriture, le style. C'est à cet aspect que nous nous intéresserons un peu plus.

\section{LE PETIT CHEZ TOUSSAINT}

Avec seulement 123 pages, La salle de bain comporte trois parties divisées en fragments numérotés. Ces fragments tiennent en un court paragraphe, souvent constitué d'une seule phrase, voire d'un seul mot. À cette brièveté fragmentaire de la narration correspondent des espaces diégétiques extrêmement restreints: la salle de bain du narrateur, évidemment, mais aussi la cuisine d'un nouvel appartement, un compartiment de train, une chambre d'hôtel, une chambre d'hôpital. Autrement dit, peu d'espaces sont évoqués, et ceux-là sont, eux-mêmes, petits. Le personnel romanesque est encore plus limité, non seulement à l'égard du nombre de personnages, mais aussi de la profondeur psychologique qui leur est accordée. D’Edmondsson (la compagne du narrateur), de Kabrowinski (l'ouvrier) et du médecin, on ne connaît ni la psyché ni même le prénom. Ils ne sont que l'objet du regard du narrateur et n'accèdent presque jamais à la parole, puisque ce dernier évite autant que possible d'engager la conversation avec eux. Le narrateur est le seul sujet du livre, mais à la différence des subjectivités fragmentées ou dédoublées que mettaient en scène les nouveaux romanciers, celle du héros de Toussaint est superficielle, sans profondeur. Son discours, livré dans un ton «impassible», est centré sur les objets autour de lui, ne laissant presque rien paraître de ses sentiments ni de sa personnalité.

Une telle réduction des composantes de la diégèse conduit à atténuer considérablement la tension narrative d'ordre romanesque, définie par Raphaël Baroni comme «le phénomène qui survient lorsque l'interprète d'un récit est encouragé à

$$
+++
$$

31 Dominique Viart, «Écrire au présent», p. 329. 32 Fieke Schoots, «L'écriture "minimaliste" », p. 127-144. 33 Jan Baetens, «Pour en finir avec la poésie dite minimaliste», p. 104.34 Fieke Schoots, "L'écriture "minimaliste" ", p. 127. 35 À tel point que de ce titre a été tirée une expression, "la génération salle de bain ", servant elle aussi d'étiquette alternative et quelque peu provocatrice pour désigner les auteurs publiés par Jérôme Lindon. 
attendre un dénouement, cette attente étant caractérisée par une anticipation teintée d'incertitude qui confère des traits passionnels à l'acte de réception ${ }^{36}$ ». Cette tension structurante, qui engendre «l'aspect dynamique» ou la «force » intrigante du roman, est absente de La salle de bain. Le déficit de profondeur psychologique des personnages empêche le lecteur de s'investir dans leur sort. Qui plus est, rien n'est «noué», pour ainsi dire, dans cette histoire: le narrateur se promène au hasard en Italie, revient à Paris, passe des journées entières dans la salle de bain, déambule dans son appartement. Presque rien de notable ou de «narrable» ne survient dans ce récit. Sans empathie pour les personnages et sans le suspense d'une narration à forte valeur événementielle, le roman offre une expérience de lecture déroutante, que la tonalité - impassible - renforce.

D'une certaine manière, la narration semble travailler contre elle-même, contre le passage du temps qu'impliquent la narrativité et le déroulement d'une intrigue. Le récit est livré en fragments, fragmentation qui s'avère artificielle. Elle hache curieusement le déroulement «normal» du temps en microséquences:

13) [...] Elle voulait faire l'amour.

14) Maintenant.

15) Faire l'amour maintenant? [... $]^{37}$

Rien ne signale ou ne justifie que la fin d'un fragment arrive, ou qu'un nouveau commence. Cette répartition postiche du récit donne l'impression d'un "pur présent ${ }^{38}$ », d'un monde où le temps recommence à zéro à chaque début de fragment. Ce temps immobile, stagnant, s'impose autant sur le plan formel que sur le plan thématique. L'obsession ${ }^{39}$ de l'immobilité du narrateur laisse paraître un effort pour restreindre tout mouvement, qu'il soit spatial ou temporel:

J'avais passé la nuit dans un compartiment de train, seul, la lumière éteinte. Immobile. Sensible au mouvement, uniquement au mouvement, au mouvement extérieur, manifeste, qui me déplaçait malgré mon immobilité, mais aussi au mouvement intérieur de mon corps qui se détruisait, mouvement imperceptible auquel je commençais à vouer une attention exclusive, qu'à toutes forces je voulais fixer. $(S B, 51)$

Le narrateur reste figé à l'intérieur du train lancé dans l'espace ${ }^{40}$; il lutte contre les mouvements à l'intérieur de son corps, cherchant ainsi à "suspendre » l'écoulement du temps dans un geste minimaliste caractéristique.

$$
+++
$$

36 Raphaël Baroni, L'œuvre du temps. Poétique de la discordance narrative, Paris, Éditions du Seuil, coll. «Poétique», 2009, p. 18. 37 Jean-Philippe Toussaint, La salle de bain, Paris, Éditions de Minuit, p. 17. Désormais, les références à cet ouvrage seront indiquées par le sigle $S B$ suivi du folio, et placées entre parenthèses dans le texte. 38 Dominique Viart, «Écrire au présent», p. 329.39 Il faut également souligner l'omniprésence de cette thématique dans Faire l'amour (Paris, Éditions de Minuit, 2002, 179 p.), du même auteur: on y trouve l'usage du mot au moins une vingtaine de fois sur 179 pages. 40 Cette scène se répète dans Faire l'amour : « Je regardais par la vitre sans penser à rien, témoin passif de cette compression de l'espace et du temps qui donne le sentiment que c'est à l'écoulement du temps qu'on assiste de la fenêtre des trains pendant que défile le paysage.» (p. 134) 
Si La salle de bain est représentative du minimalisme de Minuit sur le plan des composantes diégétiques (personnages, intrigue, temporalité) et de la tonalité impassible, la classification s'avère plus problématique pour ce qui est du style. Il suffit de relire attentivement le dernier fragment cité pour le voir. Sa première phrase est un exemple parfait d'une écriture du style minimaliste: «J'avais passé la nuit dans un compartiment de train, seul, la lumière éteinte.» Cette phrase suit la formule sujetverbe-complément, sa syntaxe et son vocabulaire sont simples, sans conjonctions de subordination ${ }^{41}$. Autrement dit, elle est d'une haute lisibilité, son sens est immédiatement accessible. Cependant, les deux phrases qui suivent troublent cette lisibilité. D'abord: «Immobile.» À première vue, on est tenté de dire qu'il n'y a rien de plus minimal qu'une phrase construite sur un seul mot. Or, si la syntaxe minimaliste implique que les phrases suivent la formule sujet-verbe-complément, cette phrase elliptique est aussi peu minimaliste que la longue phrase qui suit: le lecteur doit luimême combler le manque pour accéder à son sens. Le travail d'écriture devient encore plus évident quand on constate que ces deux phrases problématiques donnent et prennent la forme de leur contenu: «Immobile.» C'est-à-dire statique, sans flux:

Sensible au mouvement, uniquement au mouvement, au mouvement extérieur, manifeste, qui me déplaçait malgré mon immobilité, mais aussi au mouvement intérieur de mon corps qui se détruisait, mouvement imperceptible auquel je commençais à vouer une attention exclusive, qu'à toutes forces je voulais fixer.

Cette dernière phrase se dilate afin d'accueillir le mouvement, le plaisir de la répétition, les multiples conjonctions de subordination, les contournements et heurts du sens. Elle s'oppose au "petit» diégétique du compartiment sombre et à la phrase elliptique précédente. L'ensemble de ces trois phrases offre une certaine résistance à la lisibilité, mettant en opposition le simple, l'elliptique et le complexe.

Dans cette mesure, on peut dire que restreindre l'intrigue, refuser une forme de tension narrative axée sur l'événement, tout cela revient à augmenter le texte. La réduction de la diégèse et de la narrativité mène à la dilatation de la phrase, de la langue elle-même, qui s'étend afin de contenir le sens. Cette amplitude contradictoire (le petit s'oppose au grand comme l'immobilité s'oppose au mouvement ou le grave au léger) de la langue constitue, à terme, la limite haute de la lisibilité. De même, la relative brièveté du texte ne correspond pas à une pauvreté de matière, à un contenu narratif vidé d'étoffe. Une telle amplitude (de langue, de registres), mariée à la narrativité problématique, ne met pas l'accent sur le désir de raconter une histoire. L'écriture phatique ${ }^{42}$ de la "génération salle de bain " semble relever plutôt

$$
+++
$$

41 Ces traits de l'écriture minimaliste sont tirés de Fieke Schoots, "Passer en douce à la douane», p. 52-55. 42 La fonction phatique du langage a pour objet d'établir ou de prolonger la communication entre le locuteur et le destinataire sans servir à communiquer un message: "Il y a des messages qui servent essentiellement à 
d'une pulsion énonciative, d'un goût d'écrire dans une succession fragmentaire de présents que, malgré la différence de ton, l'on peut rapprocher de la saisie impressive de la réalité caractéristique des récits minimalistes québécois.

\section{«LE FEU EST LÀ, TOUT RAMASSÉ »: ANNA À LA LETTRE C DE LARRY TREMBLAY}

Anna à la lettre $C$ de Larry Tremblay est un des récits québécois que Frances Fortier et Andrée Mercier identifient comme minimaliste ${ }^{43}$. La brièveté (46 pages dans la première édition), la réduction du personnel romanesque (l'homme [sans nom], la jeune fille [Anna] et le chat), de l'espace (l'appartement d'Anna) et du temps (une après-midi d'été) font de ce récit une unité compacte, sorte de découpe brute dont les tenants et les aboutissants échappent à la compréhension. Cette unité est divisée en microséquences, chacune d'elles se trouvant isolée de la suivante par un espace blanc et un retour de page qui manifeste le morcellement de la narration. Bien qu'entrecoupé d'ellipses, le récit reste successif: il raconte, dans l'ordre, les gestes, les déplacements, les propos ou les pensées des deux personnages principaux en faisant alterner le point de vue de l'un à l'autre. On a donc non pas, comme chez Toussaint, une voix narrative subjectivement assumée, mais une oscillation constante de la focalisation sans ancrage énonciatif fixe. La progression à la fois successive, fragmentaire et elliptique caractérise la conduite minimale de ce récit, qui aligne les microséquences en l'absence d'une perspective d'ensemble. Aucune intrigue, aucun préalable n'oriente cette histoire qui relate une suite de faits et gestes d'ordre infra-événementiel sans origine ni devenir, que le quotidien exemplifie. Les faits d'ouvrir le frigo, de préparer un thé glacé, d'aller sur le balcon ou d'allumer une cigarette sont racontés par le menu, produisant l'effet d'une lenteur extrême. L'image des femmes et des hommes faisant du taï-chi dans le parc condense bien cet étrange ballet qui s'étire sans que l'on sache d'où cela vient ni où cela mène. L'extériorité de la vision, qui décrit en détail et en surface, sans motivation psychologique, les gestes des personnages, épouse en partie la vision distanciée du réel assumée, chez Toussaint, par un narrateur déconnecté d'une réalité omniprésente ${ }^{44}$. C'est d'abord

$$
++
$$

établir, prolonger ou interrompre la communication, à vérifier si le circuit fonctionne ("Allo, vous m'entendez?"), à attirer l'attention de l'interlocuteur ou à s'assurer qu'elle ne se relâche pas ("Dites, vous m'écoutez ?") ou, en style shakespearien, "Prêtez-moi l'oreille!" et à l'autre bout du fil, "Hm-hm!" » (Roman Jakobson, Essais de linguistique générale, t. 1, Paris, Éditions de Minuit, 1963, p. 217). 43 Frances Fortier et Andrée Mercier, «La narration du sensible dans le récit contemporain". 44 Cette distanciation est, chez Toussaint, de l'ordre de l'oxymore: la subjectivité devient le lieu d'une désaffection paradoxalement esthétique; le spectacle du monde en évacue le drame sur un mode drolatique très éloigné de l'atmosphère orageuse qui règne dans l'univers d'Anna à la lettre C. À noter cependant que si la tension dramatique est plus palpable chez Tremblay, plus directement reliée aux affects des deux personnages, la «dégaine» impassible de l'instance narrative chez Toussaint est elle aussi le véhicule d'affects qui, aussi détonants et fulgurants soient-ils, n'en chargent pas moins le récit d'émotions. Cela est encore plus manifeste dans la récente trilogie de Toussaint, mais apparaît dès La salle de bain. La discontinuité des marques affectives ne permet cependant pas, dans ce roman, d'ordonner les événements suivant une logique du sensible. 
par ponctions, de manière aussi fulgurante qu'inexpliquée, que le désir de l'homme et le dégoût d'Anna émergent, creusant, en deçà des gestes et des mots anodins, une tension affective. S'ils restent attachés aux corps, ces affects investissent également l'intériorité des personnages sans lui donner préséance: la logique de l'action n'est pas ici reversée dans des univers mentaux capables d'orienter l'histoire du dedans. Au contraire, on oscille sans cesse entre univers extérieur et monde intérieur (souvenirs, émotions et pensées d'Anna, désirs et pensées de l'homme), sans surplomb. Une esthétique filmique se dégage d'un tel mode narratif dont l'effet n'est pas, comme chez Toussaint, celui d'une impassibilité ${ }^{45}$. Car si la puissance visuelle génère une lenteur capable de suspendre l'orientation et la signification des faits et gestes, elle crée aussi une tension dramatique forte, véritable suspense qui donne aux affects latents, informulés (intérieurs), une grande portée structurante.

Cela passe aussi par un jeu de figuration disséminé mais constant, chargeant les sensations, les gestes et les silences d'une intensité affective et dramatique tangible. Pas à pas, en l'absence d'une trame psychologique, un climat s'affermit. Il sature l'atmosphère d'une potentialité qui, au fil des reprises, finit par dominer. La prise en relais des figures contribue à étirer le suspense qui, dans la seule mesure où il pourrait arriver quelque chose, charge tout incident d'une tension romanesque palpable. Lieu, gestes, objets, personnages sont également affectés: «Elle la [l'allumette] place sous les yeux de l'homme [...]. Le feu est là, tout ramassé. Ressort chimique, bête aux aguets prête à se lancer dans l'espace. [...] De nouveau, le silence flambe ${ }^{46}$ »; "Comme si leur corps, figé dans la position de départ, guettait le signal de la course. » $(A L C, 30)$ Là où, chez Toussaint, s'installe une immobilité dynamique ${ }^{47}$, chez Tremblay, c'est un élan arrêté qui caractérise le récit à travers un réseau de figures qui renforcent l'expression répétée du désir de l'homme ${ }^{48}$. Ainsi, graduellement, dans la succession des descriptions d'une quotidienneté saisie au ras des gestes et de son retentissement sensoriel et affectif (figuré ou non), le récit s'infléchit vers une fin, à savoir l'étreinte de l'homme comme «passage à l'acte». Mais cette étreinte est interrompue par une crise d'indigestion, accident trivial, à la limite du grotesque, qui met un terme à la rencontre sans qu'Anna ait pu réagir: elle reste paralysée, «bloquée» à la lettre C. La crise fauche toute possibilité pour elle d'affirmer (ou pas) un agir. En ce sens, l'étreinte ruine la logique de l'action qu'elle semble pourtant servir. Le récit est bouclé sur la réitération de l'échec qui flottait dans

$$
+++
$$

45 Pour une analyse plus fouillée du rapport au cinéma, voir Marie-Pascale Huglo, «Anna à la lettre C de Larry Tremblay. Une esthétique filmique», Héliane Ventura et Philippe Mottet (dir.), Pratiques de la transgression dans la littérature et les arts visuels, Québec, L'instant même, 2009, p. 113-126. 46 Larry Tremblay, Anna à la lettre C, Montréal, Les Herbes rouges, 1992, p. 11. Désormais, les références à cet ouvrage seront indiquées par le sigle ALC suivi du folio, et placées entre parenthèses dans le texte. 47 «La peinture, en général, n'est jamais immobile. Comme aux échecs, son immobilité est dynamique. Chaque pièce, puissance immobile, est un mouvement en puissance.» $(S B, 84)$ Nous reviendrons sur ce point. 48 La reprise des manifestations de ce désir est aussi une manière elliptique d'orienter le récit et d'installer la série des événements dans une durée narrative: «L'homme n'a plus qu'un désir : goûter à la sueur d'Anna. » (ALC, 17); «L'homme a faim. Aussi. » (ALC, 21; nous soulignons.); "L'homme ne se cache pas qu'il mangerait bien dans la bouche d'Anna.» (ALC, 25); «L'homme entend un bruit d'eau. Il s'entend dire: "Je boirais son urine." (ALC, 27); «L'homme sait maintenant ce qu'il veut d'elle.» (ALC, 38; nous soulignons.) 
l'humidité ambiante, faisant du motif récurrent du silence non plus l'expression d'affects indicibles, mais l'expansion désormais sans appel du ratage qui couvait ${ }^{49}$ : «Il y a un silence qui ne peut être rien d'autre que le gonflement du sale, du triste, de l'échoué.» $(A L C, 53)$ Ce silence, que le style elliptique à la fois reconduit et amplifie (le non-dit étant aussi - on l'a vu avec Toussaint - un appel à la coopération du lecteur), exemplifie bien le minimalisme de Tremblay combinant une suite de gestes décrits sans perspective ni conséquence et une dissémination de sensations intérieures, de motifs elliptiques et de figures expressives qui fabriquent une pure potentialité. Fort d'une narrativité toute romanesque (à laquelle les topoï de la rencontre entre un homme et une femme et de l'orage contribuent), le récit cultive un suspense qui ne vit que pour repousser l'action: le fait qu'il soit bouclé une fois accompli le seul geste décisif de cette histoire, à savoir l'étreinte, sans qu'il ne soit «rien arrivé» - l'orage n'éclate pas; l'homme et Anna se retrouvent en quelque sorte à la case de départ, les possibles en moins - , souligne bien l'échec de l'action. $C^{\prime}$ est donc en gardant en réserve, en tension, la possibilité de l'action que le récit tire, du sein de sa lenteur même, une force dynamique "prête à se lancer dans l'espace» $(A L C, 11)$, jusqu'à l'étouffement final.

\section{"COMME DANS UN FILM». \\ LE BRUIT DES CHOSES VIVANTES D'ÉLISE TURCOTTE}

Du côté d'Élise Turcotte, on peut se demander jusqu'à quel point le «mode mineur» recoupe le minimalisme narratif. On retrouve, dans Le bruit des choses vivantes, la réduction spatio-temporelle (une année dans la vie d'une mère, Albanie, et de sa fille, Maria, ancrée dans l'espace domestique) et celle du personnel romanesque (on ne sort pas du voisinage, même si celui-ci s'agrandit), caractéristiques de la réduction minimaliste ${ }^{50}$. La narration, assumée au je, est dispersée au fil des instants, ce qui coupe court à une perspective d'ensemble. Ce mode fragmentaire transforme la conduite du récit, saisi dans des moments discontinus mais successifs, dans lesquels le non-dit occupe une grande place. On a là une caractéristique commune aux cas qui nous occupent malgré une différence de tonalité importante: la voix narrative chez Toussaint est distanciée, parfois facétieuse; elle se montre plus grave chez Tremblay (malgré son impersonnalité), gravité également à l'œuvre dans Le bruit des choses vivantes, où la subjectivité ne se présente pas comme impassible, bien au contraire. Le retentissement dramatique et affectif s'empare de toute chose sur un mode elliptique, moitié factuel, moitié figuratif : «Les jours passent et rien n’est plus jamais pareil $[\ldots]$ il est toujours trop tard pour une chose ou une autre ${ }^{51}$.» Le drame de ce

$$
++
$$

49 Le ratage apparaît au début du récit, il flotte dans l'atmosphère: «L'humidité du moment donne au salon l'odeur de l'échec.» $(A L C, 9) \quad 50$ Cette lecture s'inscrit dans le prolongement de l'article de Marie-Pascale Huglo sur ce roman, "Le quotidien en mode mineur: Le bruit des choses vivantes d'Élise Turcotte», Voix et Images, vol. XXXIV, n 3, printemps-été 2009, p. 99-115. 51 Élise Turcotte, Le bruit des choses vivantes, Montréal, Leméac, coll. «Babel», 1991, p. 54. Désormais, les références à cet ouvrage seront indiquées par le sigle $B C V$ suivi du folio, et placées entre parenthèses dans le texte. 
qui disparaît, de ce qui meurt, se fait à la fois insistant (les jamais et toujours radicalisent l'affirmation) et élusif (la généralité désigne tout et rien à la fois), passant du concret à l'abstrait (du particulier au général) avec aisance. Même les faits concrets sont transfigurés: «Maria [...] a fait un bricolage géant avec des feuilles d'érable, du pop-corn et plein d'autres trésors.» (BCV, 51; nous soulignons.) L'image du trésor, qui épouse l'émerveillement de l'enfant, évacue toute trivialité du quotidien, de sorte que l'arrimage entre le concret et l'abstrait ne verse pas dans le drolatique (Toussaint) ou le drame frôlant parfois la farce (Tremblay), mais élève le propos tout en alimentant la quête de sens.

Car Albanie cherche à trouver un sens à ce qui se disloque et toujours disparaît, donnant ainsi cohérence et motivation aux instants les plus anodins qui finissent, par son entremise, par être reliés. Une trame à la fois psychologique et dramatique se met en place, conjuguant histoire d'amour, chronique domestique, drame familial et enquête policière. Une intrigue romanesque multiple émerge du kaléidoscope du quotidien. À partir de là, les incidents non seulement se relient, mais s'ordonnent, s'enchaînent, entraînent des conséquences: que Félix, voisin de Maria, fasse tout seul, à trois ans, le trajet de sa maison jusqu'à la garderie est un incident d'importance majeure. En ce sens, même s'il s'agit d'un incident infime, son retentissement intime et sa capacité à déclencher l'action ("Lise a dit, maintenant c'est à moi d'agir. » [BCV, 133]) n'ont rien de minimaliste. Même chose pour Maria, qui évolue en fonction d'événements minuscules qui font signe, se détachent, deviennent déterminants: «Depuis qu'elle sait que je l'ai vue de ma fenêtre, Maria n'a plus tellement peur à la garderie.» $(B C V, 123)$ L'événement fait des expériences et de leur répercussion affective un agent de transformation. Le ressenti mobilise ainsi une narrativité chevillée à l'action en devenir. Celle-ci se manifeste certes sur un mode lacunaire, subjectif, intériorisé, elliptique, discontinu, mais ne suspend pas la portée ni le sens de ce qui arrive et change. La vision prégnante et les nombreuses allusions au cinéma sont une manière d'investir les images d'une narrativité potentielle ancrée dans la mémoire collective. Si, comme chez Tremblay et Toussaint, la potentialité «romanesque» passe entre autres par le répertoire cinématographique, si elle permet de mettre les instants sous tension et d'intégrer l'imaginaire de l'aventure (de l'amour, du voyage, du mystère...) aux vignettes du quotidien, elle contribue aussi, chez Turcotte, à la progression de l'histoire. Ainsi en est-il à propos d'Alexandre, l'amant avec qui rien ne pourra arriver: "Je dis que nous ne sommes pas dans un film d'amour ici. Il n'y a pas d'unique chambre avec un lit immense et des fenêtres ouvertes. " $(B C V, 47)$ Le cinéma de la première rencontre avec Pierre s'avère, lui, d'emblée décisif: « Juste au moment où il referme la porte derrière lui, Maria et moi sortons et, comme dans un film, deux portes se ferment en même temps, deux regards se croisent et le premier geste que l'on fait est alors immanquablement dirigé vers l'autre personne. » (BCV, 162) Immanquablement, cette rencontre aura une suite : la coïncidence "veut dire" quelque chose. Les histoires qui prennent forme restent allusives, elles se fondent dans la portée symbolique - figurative - des choses bien plus que dans une quelconque facticité. Elles s'enchaînent à travers un réseau de motifs qui se répondent et se prolongent, par résonance intérieure plutôt que par péripéties. Cependant, 
péripéties et «coïncidences ${ }^{52}$ » investissent de l'intérieur les moindres incidents, elles les transforment en signes du hasard ou du destin, donnant à la petite vie ce souffle romanesque "pas croyable» $(B C V, 202)$ dont le cinéma est porteur. Le bruit des choses vivantes se démarque en cela des "grands romans» remplis d'aventures, il raconte sur un "mode mineur» plus réfléchi, intériorisé, suggestif, mais il ne renonce pas à l'action pour autant et incorpore l'aventure dans les minutes du quotidien. Parce que des événements signifiants se détachent, trouvent leur place dans une histoire et font «leur cinéma», on ne peut véritablement parler ici de narrativité minimaliste. La logique du sensible et l'esthétique visuelle ne suspendent pas l'action comme chez Tremblay ni ne la neutralisent comme chez Toussaint : elles la sous-tendent et la reconduisent à leur manière.

\section{NARRATIVITÉ MINIMALISTE, OU «L'IMMOBILITÉ DYNAMIQUE »}

À l'issue de cet aperçu, il ressort que c'est à partir du moment où une tension s'instaure entre une série d'incidents et le sens de cette série que l'on peut considérer la narrativité comme minimaliste: un fossé se creuse entre les faits relatés et leur portée significative sur le plan de l'action, des affects ou du savoir. L'amplification de la vision coïncide non pas avec l'élimination ou le brouillage chronologique mais avec la suspension du sens de cette (ces) suite(s), de ce que «cela veut dire». Chez Turcotte, par contre, "ce que cela veut dire» se trouve au centre de la quête d'Albanie, qui interroge et sonde toute chose pour en tirer le cœur signifiant, raison pour laquelle, en dépit de l'omniprésence d'impressions visuelles sensibles, nous ne considérons pas son roman comme minimaliste ${ }^{53}$.

Le réseau des motifs, très présent dans le roman de Turcotte, doit être replacé dans une dynamique narrative d'ensemble: loin de contribuer à une cohérence problématique opposant une résistance, fût-elle passive et momentanée, à l'ordre du sens, la récursivité des motifs permet, dans Le bruit des choses vivantes, de recharger symboliquement l'univers domestique. La cohérence discontinue qui s'instaure rejoint finalement l'ordre du récit plutôt que de le déstabiliser ou le suspendre. En cela, le mode mineur n'est pas plus minimaliste que le minimalisme n'est un mode mineur (un amoindrissement) du roman. Le minimalisme n'atteint pas au premier chef l'amplitude factuelle d'une histoire (on voyage beaucoup, parfois très loin, dans les romans de Toussaint) ni l'émergence d'événements, mais il ébranle l'ordre logique établissant une sorte d'axiologie événementielle qui permet, justement, de faire la part de l'important et du mineur. C'est cela qui est mis à mal dans une sorte de déflation généralisée. Dans cette mesure, l'absence d'enchaînement causal, relevée par

52 «La vie est une coïncidence rare.» $(B C V, 181) \quad 53$ Notons que la propension à l'abstraction, présente dans les trois récits retenus, peut aussi bien renforcer l'arrimage problématique entre la pensée et la réalité, manifeste dans l'écart entre les questionnements métaphysiques et le monde concret (Toussaint, Tremblay), que le résoudre dans une conciliation existentielle (Turcotte). 
maints critiques, est déterminante. Elle se combine souvent avec une fragmentation, une absence de perspective et une esthétique visuelle - voire filmique - marquée. Les incidents se découpent, les gestes et les scènes se suivent sans que l'on sache quelle en est la raison, l'importance ${ }^{54}$. Cela passe aussi par un clivage marqué, chez Toussaint, entre le ton (léger, distancié, impassible) et les événements racontés qui, à l'occasion, ne manquent pas de relief. Pourquoi raconter ceci plutôt que cela et où cela mène-t-il ? Telle est la question que soulèvent les romans minimalistes, bousculant nos attentes narratives, y compris lorsqu'il «arrive» quelque chose ${ }^{55}$. La pertinence de l'histoire racontée est déçue jusque dans l'extraordinaire, d'où l'idée d'une superficialité attachée à ce courant. Là où le minimalisme perturbe l'ordre narratif du sens en bousculant, à différents niveaux, la hiérarchie du majeur et du mineur, le mode mineur valorise le petit, il en établit le sens et - fût-ce en pointillés - le récit.

Pour tenter de replacer l'esthétique minimaliste dans un geste global, disons alors qu'elle relève d'une relation conflictuelle entre le régime narratif privilégiant le sens (l'intrigue) et le régime esthétique privilégiant l'apparaître. Pour le dire avec Rancière, une tension s'instaure, au sein du récit, entre muthos et opsis, entre «matière fictionnelle» et "matière sensible ${ }^{56}$ ", le sensible relevant ici moins du pathos que du spectacle capable de «suspendre " (d'étaler, d'immobiliser) le sens, ce qui arrive (actions, sensations, émotions) se trouvant mêlé à ce qui apparaît ${ }^{57}$. Si, du côté des écrivains de Minuit, l'esthétique passe par des dichotomies tonales aussi marquées que distinctives, il n'en demeure pas moins que cette conflictualité entre esthétique visuelle de l'apparaître et intrigue du sens ressort tant en France qu'au Québec. La comparaison entre les deux courants et leur critique montre d'ailleurs que la saisie impressive, subjective, du réel ne dessert pas cette esthétique mais s'y greffe à partir du moment où les sensations se dissocient du sens. Si nous ne faisons pas des marques de la subjectivité un critère distinctif, l'insistance de la critique québécoise là-dessus nous invite à reconsidérer l'importance d'une telle «saisie impressive» dans les romans de Minuit, dont l'impassibilité affichée n'est pas pour autant dépourvue d'affects ${ }^{58}$. Si nous tirons la logique du sensible, relevée par

$$
++
$$

54 Si le quotidien participe souvent de l'esthétique minimaliste avec les gestes et les incidents insignifiants, sans portée décisive, qui le composent, il ne constitue pas un critère déterminant. 55 Le décalage qui s'instaure, chez Toussaint, entre la fléchette qu'il plante dans le front d'Edmondsson, ensuite conduite à l'hôpital, et l'absence de motivation psychologique manifeste qui diffracte cet événement dans une succession fragmentaire d'images et de gestes oppose ainsi au potentiel éminemment dramatique de l'incident son traitement esthétique "superficiel», axé sur une visualité minutieuse à l'extrême, et l'absence de conséquence que la fragmentation, "coupant court», sert bien (la deuxième partie se clôt sur cette image: «Nous nous embrassâmes dans le couloir blanc. » [SB, 91]). 56 Jacques Rancière, «Une fable contrariée», La fable cinématographique, Paris, Éditions du Seuil, coll. «La librairie du Xxi ${ }^{\text {e }}$ siècle», 2001, p. 7-28. 57 Le couplage entre une esthétique visuelle/ sensible et un sens narratif problématique se distingue bien de l'association entre une «diégèse [...] de type minimaliste» (p. 213) et une amplification énonciative dans les récits «logodynamiques». Ces romans, dont l'histoire est réduite et l'énonciation, amplifiée, ne sont pas minimalistes puisque «le moindre fait, le moindre geste comme le moindre mot [...] peuvent se trouver élevés à la hauteur du récit» (p. 213). Le verbe porte et emporte le récit, dont le sens, ainsi «rehaussé», n'est pas suspendu ni mis à plat. (Elisabeth Haghebaert, "Une narrativité logodynamique tendance destroy: les récents Ducharme», René Audet et Andrée Mercier [dir.], La littérature et ses enjeux narratifs, p. 203-229). 58 Nous ne donnerons qu'un exemple dans lequel la séparation entre le narrateur et Edmondsson passe par la figuration d'un déchirement dont le retentissement «sensible » est, 
Fortier et Mercier, du côté de l'esthétique, c'est pour l'infléchir et l'amplifier. Enfin, les contradictions de la critique relativement à la marginalisation de la littérature dans un monde saturé par les médias, au soupçon de la représentation doublé d'une surenchère des renvois aux œuvres et aux médias, trouvent dans cette esthétique narrative (au sens fort, conflictuel, du terme) leur place. L'apparition du monde, avec la puissance visuelle qui s'y rattache, va de pair avec une prise de conscience et, parfois, un jeu sur les moyens de la représentation: le réel n'apparaît pas en dehors des médias qui le font apparaître tel. Les jeux intertextuels, le deuxième degré (écrivains de Minuit) et les nombreux renvois aux médias de l'image comme à la littérature visent non pas à rompre avec «l'illusion de la mimèsis » mais, plutôt, à en démultiplier les modes, voire les artifices, sans naïveté certes, mais sans non plus renoncer aux forces de l'imaginaire. Le constat désenchanté d'une littérature de l'après, poussée dans les marges, sous-estime le parti qu'elle tire des médiations démultipliées du réel.

La narrativité minimaliste ne se ramène à une absence de matière que si l'on évacue la tension narrative qu'elle génère du sein du conflit qui l'anime. L'absence d'intrigue ne correspond pas, somme toute, à une absence de tension, de suspense. L'orage qui couve, chez Tremblay, montre à quel point la quasi-absence d'action est porteuse d'un imaginaire dramatique procurant un potentiel explosif à la moindre tête d'allumette. Chez Turcotte, la domesticité se trouve réinvestie de l'intérieur par des scénarios romanesques, merveilleux, qui enchantent le quotidien. Chez Toussaint enfin, alors que le narrateur s'évertue à immobiliser le cours du temps et à aplanir tout conflit, les remous contradictoires - angoissants - de sa quête investissent le roman ${ }^{59}$. Même en l'absence d'enchaînement, la réticence à faire du comportement énigmatique du narrateur une intrigue psychologique génère une tension heuristique, un désir de savoir. Pressante, oppressante dans certains fragments, la tension mobilise cette histoire en l'absence d'intrigue et - presque - d'évolution, poussant ainsi à bout le paradoxe tragicomique d'une immobilité dynamique ${ }^{60}$. La tension narrative, pétrie d'imaginaires romanesques, filmiques et autres, est un des aspects

$$
+++
$$

à l'instar de l'atmosphère orageuse chez Tremblay, palpable: "Sur le quai, devant la porte ouverte du compartiment, je voulus la serrer dans mes bras; elle me repoussa avec douceur. Les portes furent claquées une par une. Et le train est parti comme un vêtement se déchire.» (SB, 95) 59 Le narrateur apprécie chez Mondrian «l'immobilité immobile», c'est-à-dire "l'absence de toute perspective de mouvement» $(S B, 84)$, mais s'il tend vers une telle suspension, tente de s'en approcher le plus possible, sa quête se solde par un retour au mouvement (le roman se termine avec sa sortie de la salle de bain) et elle est ponctuée par une série de réflexions marquant son caractère impossible. Certaines images vont dans le même sens, véhiculant aussi une angoisse diffuse mais sensible qui génère, par ponctions, une tension narrative: «Lorsque, le matin, je me réveillais, je voyais la journée à venir comme une mer sombre derrière mes yeux fermés, une mer infinie, irrémissiblement figée. » (SB, 86) La contraction de l'à venir et du figé crée une sorte de tension immobile chargée d'imaginaire que le roman, certes, ne déploie pas, mais qui revient sans cesse. Cela sous-tend un dialogue avec Pascal, du reste cité dans $L a$ salle de bain, dialogue qu'on ne saurait réduire à un second degré ludique: ce serait évacuer toute la tension, l'angoisse, le malheur "de ne savoir pas demeurer en repos, dans une chambre » (Blaise Pascal, «fragment 126 », Pensées, Michel Le Guern [éd.], Paris, Gallimard, coll. «Folio classique», 2008 [1995], p.118). 60 La formule de Toussaint exemplifie la narrativité minimaliste: à l'absence de mobilisation romanesque et à l'impression que, malgré les incidents et déplacements de surface, le récit "n'avance pas», correspond un univers de possibles chargés d'imaginaires qui, à eux seuls, mobilisent une narrativité latente, "tensive», très active. 
sur lesquels la critique a le moins insisté. C'est pourtant elle qui procure à l'esthétique minimaliste une narrativité agissante qui puise, à même l'ordre du récit qu'elle combat, ses forces vives. 